\title{
DISCLAIMER.
}

This report was prepared as an account of work sponsored by an agency of the United States Government. Neither the United States Government nor any agency thereof, nor any of their employees, makes any warranty, express or implied, or assumes any legal liability or responsibility for the accuracy, completeness, or usefulness of any information, apparatus, product, or process disclosed, or represents that its use would not infringe privately owned rights. Reference herein to any specific commersial product, process, or service by trade name, trademark. manufacturer, or otherwise does not necessarily constitute or imply its endorsement, recommendation, or favoring by the United States Government or any agency therenf. The views and opinions of authors expressed herein do not necessarily atate or reflect those of the United States Government or any agency thereof.

\section{ADVANCED SCIENTIFIC COMPUTING ENVIRONMENT TEAM (U)}

by

\section{J. P. Church}

Westinghouse Savannah River Company

Savannah River Site

Aiken, South Carolina 29808

A paper proposed for publication in the

Appendix to Martin Marietta Energy Systems and DOE technical report

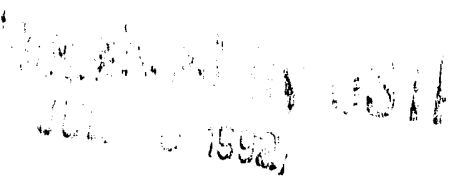

The information contained in this abstract was developed during the course of work done under Contract No. DE-AC09-89SR 18035 with the U.S. Department of Energy. By acceptance of this paper the publisher and/or recipient acknowledges the U.S. Government's right to retain a nonexclusive, royalty-free license in and to any copyright covering this paper, along with the right to reproduce and to authorize others to reproduce all or part of the copyrighted paper.

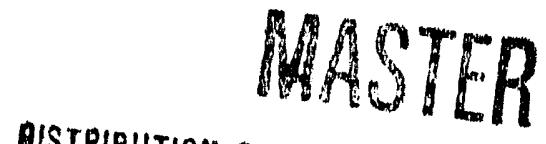




\title{
ADVANCED SCIENTIFIC COMPUTING ENVIRONMENT TEAM
}

\author{
J. P. Church, Advisory Engineer \\ Scientific Computations Section, Savannah River Technology Center \\ Westinghouse Savannah River Company, Aiken, SC 29808
}

\section{OBJECTIVE}

The mission of the ASCENT (Advanced Scientific Computing EnvironmenI) Team is to continually keep pace with, evaluate, and select emerging computing technologies to define and implement prototypic scientific computing environments that maximize the ability of scientists and engineers to manage scientific data. These environments are to be implemented in a manner consistent with the site computing architecture and standards and strategic plans for scientific computing.

A broad, long term, goal of the ASCENT Team is to provide a computing environment that will let scientists and engineers function at the higher level of abstraction that is their actual area of technical expertise. The scientist/engineer should be able to solve problems by interacting with conceptual representations drawn directly from the scientific and engineering domains. In this environment the scientist/engineer (i.e., the "problem solver") builds the problem model with reusable virtual objects having associated attributes and behaviors, including any real or artificial constraints. The problem solver could then test the model by perturbing it interactively and observing quantitative (archived experimental measurements; simulated or computed data) and/or qualitative (trends, approximations) responses. Such an environment would greatly aid the solution and understanding of scientific and engineering problems.

Some specific examples may help clarify these ideas. A thermalhydraulics analyst should be able to interact with his ${ }^{1}$ desktop terminal to build a RELAP model of an SRS reactor by dragging icons of pumps, pipes, vessels, heat structures, etc., to assemble the completed model. The icons would contain the data, correlations, relationships, physics, etc. pertaining to the actual object they represent. The analyst could then specify a flow transient that reproduces the flow changes imposed during an actual reactor test, compute the results, call up the archived experimental results and display them alongside the computed results, execute other approximate miodels for the same conditions (e.g., FLOOD code), and display those results to compare with the RELAP and experimental values. The analyst should be able to easily compute correlations and then display and compare trends of both experimental and computed results. Similar analyses could be performed using various reactor physics and charge design codes, Reactor Monitoring System data or Control Computer data, and simulator response.

Another example might be an engineer who needs to know the location of the safety rods in one of the SRS reactors. He would be able to interact with his desktop terminal to request a representation of a reactor facemap, choose a pull-down menu to select safety rods, and request a printout and/or screen display of the X-Y coordinates of all, or any subset of, the safety rods. If the engineer were analyzing the risk of safety rod

1 Throughout this document the use of words denoting gender is for convenience only. The reader should substitute the concept of "the problem solver" in such usage. 
failure, he could select a number of rods from the facemap display, either singly or by drawing a shape around a group, and choose a pull-down menu to indicate that those rods were assigned 'inoperable' condition. The engineer could then use screen icons representing computer codes and databases to build a procedure that would determine the reactivity worth of the perturbed safety rod complement, and, depending upon the series of codes and recursions specified, could complete the probabilistic analysis of risk. The engineer could interrogate the meteorological database, display a windrose for each stability class, use the mouse to select and perturb a portion of any windrose, and re-do the analysis. Alternatively, the engineer could interrogate the real-time wind data being gathered by the onsite weather towers, and examine alternate evacuation plans by selecting highways with associated attributes of carrying capacity (population source depletion capacity) to minimize both population and maximum individual dose following a postulated major core melt accident.

\section{PROPOSED SYSTEM}

The major trends in computing hardware and software technology clearly indicate that the future "computer" will be a network environment that comprises supercomputers, graphics boxes, mainframes, clusters, workstations, terminals, and microcomputers (i.e., a full complement of clients and servers). This "network computer" will have an architecturally transparent operating system allowing the applications code to run on any box(es) supplying the required computing resources (e.g., cycles, storage). The environment will include a distributed database and database managing system(s) that permits use of relational, hierarchical, object oriented, GIS, et al, databases.

The benefit of this proposed environment is that it will provide full flexibility to take advantage of the latest advances in hardware and software and, at the same time, maximize the ability to process scientific information and minimize the time required to develop products. And the newly hired S/E will be able to contribute much more quickly to SRS research and development programs.

\section{PROGRAM}

To reach this long term goal we have implemented a stepwise progression from the present assemblage of monolithic applications codes running on disparate hardware platforms and operating systems.

The initial components of this program include:

- Development of a prototype distributed computing system based on Unix, $X$ Windows, network computing hardware (heterogeneous environment), distributed databases, and distributed file systems.

- Development of portable graphics tools

- Initiation of training to implement and disseminate above tools and methods.

The first item and second items are well underway with significant new capabilities already provided to the applications community onsite. Portable graphics tools are being developed using used Object Oriented Technology concepts and are the 
subject of this Appendix. More complete information about our program has been published in external reports.2,3

\section{GRAPHICS TOQLS AND APPLICATIONS}

As discussed above, the ASCENT Team is developing a core set of graphics tools to be used in the scientific computing arena at SRS. These tools are intended to make application development easier, human interfaces more intuitive, and application codes more portable by separating the calculations from their input and output. The graphics tools are being developed using industry standards such as the $\mathrm{C}$ Language, $X$ Window System, X Toolkit, Motif ${ }^{\mathrm{TM}}$ Graphic Tool Kit, and Unix. Each tool is intended to be the standard graphical user interface for the site and to provide the capability for applications output to be viewed from anywhere onsite. The tools are being developed using Object Oriented Technology concepts.

The reactor facemap tool is discussed next. This tool was used in the subsequent development of a Reactor Monitoring System application (to display online reactor data) and a Graphic Reactor FaceMap function (to create input for charge design) which are also discussed below.

\section{IV.1 FaceMap Tool}

The first graphic tool completed, FaceMap, displays a reactor facemap4. The FaceMap tool has been tested with certified Senior Reactor Operators to identify 'human engineering discrepancies'. The prototype was developed using the Motif ${ }^{\mathrm{TM}}$ Graphic Tool Kit, but the production version of the tool was written as a widget based on the Xtoolkit Intrinsics. This facilitated packaging the tool as a separate reusiable entity that is distinct from the application.

\section{IV.1.1 EaceMap Features}

The FaceMap tool provides 'pointer tracking' and 'enter notify' capabilities. 'Pointer tracking' places a crosshair on the facemap at the center of the hex that the mouse pointer is in. The crosshair runs the length and width of the facemap. This makes it easier to determine which $x, y$ location the pointer is in. Pointer tracking also highlights the particular hex by drawing a line around the outside of the hex. 'Enter notify' notifies the application when the mouse pointer moves to a new hex position. An example of a use of this feature would be an application which displays the online computer number corresponding to the position of the pointer.

The tool can outline portions of the reactor facemap (positions, clusters, gangs, sectors and systems), display the facemap in grayscale as well as color, and produce

2 J. P. Church. "Advanced Scientific Computing Environment Group-New Scientific Database Management Task-Program Plan (U)". WSRC-TR-91-70 (February, 1991).

3 J. P. Church. "Progress Report: Advanced Scientific Computing Environment Group-New Scientific Database Management Task (U)". WSRC-TR-91-420 (June, 1991).

4 J. C. Roberts. "Interactive Graphical Reactor Facemap Tool. Patent Disclosure No. SRS-91-230 (May 23, 1991). 
PostScript output for printing the facemap. The facemap will also print on an inexpensive black-\&-white PostScript printer and will simulate grayscale.

Presently, the FacelMap tool runs on IBM RS/6000, DEC RISC, HP 700, and Sun SPARC workstations running their individual versions of Unix; VAX systems operating with VMS; and a Mac Ilci with AVUX (the Mac $X$ Window System must also be installed). FaceMap can be displayed on any box (including Mac's and PC's) having $X$ server software.

\section{IV.2 FaceMap Tool Applications}

\section{IV.2.1 Beactor Monitoring System}

The Reactor Monitoring System (RMS) facilitates monitoring and interpreting reactor operation by providing for collection, storage, and retrieval of reactor operating data. The FaceMap-RMS application prototype provides a full graphic display of a facemap of temperatures, flows, powers, or any other dimension of the reactor assemblies. This application was extended to display a sequence of historical data at various frame rates. The result is that the display of online reactor data can be viewed from anywhere on the local area network.

\section{IV.2.2 Graphic Reactor FaceMap (FM)Function}

The latest FaceMap tool application developed is intended to replace the text-based RM function used to facilitate data input to a reactor charge design code. The RM function creates an image of a reactor facemap during charge design. That image is then processed to point to desired data records to describe a specific reactor charge for physics codes calculations. The text-based RM function is a pseudo-graphic that is difficult to use, imposes unnecessary constraints on the user, and is prone to user error.

The new graphics function, named FM, replaces the RM function. Although the RM function will continue to be available, it is expected that user productivity obtained with the new FM function will be so greatly increased that a user will be able to set up a charge design much more rapidly, by a factor of 10-20 for simpie problems and as much as 100 times faster for very complicated problems, than previously possible. Early tests show that goal is achievable.

The new function permits multiple axial-level reactor maps, creation of assembly types through an assembly editor, mapping between 2-character mnemonic label and corresponding reactor input data record, cutting and pasting between axial levels, and cutting and pasting of positions, clusters, sectors, systems and gangs.

The new FM function has five panels controlled by the user: (1) FaceMap Panel to display the facemap and status information; (2) Assembly Palette Panel to display a list of the current assemblies that can be put into positions on the facemap; (3) Assembly Editor Panel to create assemblies by specifying a name, type, mnemonic, color, and GLASS record; (4) Preferences Panel to specify if boundaries (gang,sector, or system) are to be shown, if hexes are to be outlined, if assemblies are to be labeled (OLC, 
$\mathrm{mn}$ ?monic, or type), if the pointer is to be to tracked with crosshairs and hex high lighting, etc.; (5) Selector Panel to identify specific groupings of reactor positions.

These panels are discussed more fully below.

IV.2.2.1

Face Map Panel

The FaceMap Panel, shown in Figure 1, is the main working area for the FM function. It is used to display the current charge, select positions and it contains the application menus. Shown in Figure 1 is a fictitious example charge with the options menu activated. When a user presses the mouse button on a particular position, that position becomes selected. A selected position will have a white outline around it. Once the position is selected the user may place an assembly into that position or perform an Editing function such as Cut, Copy and Paste.

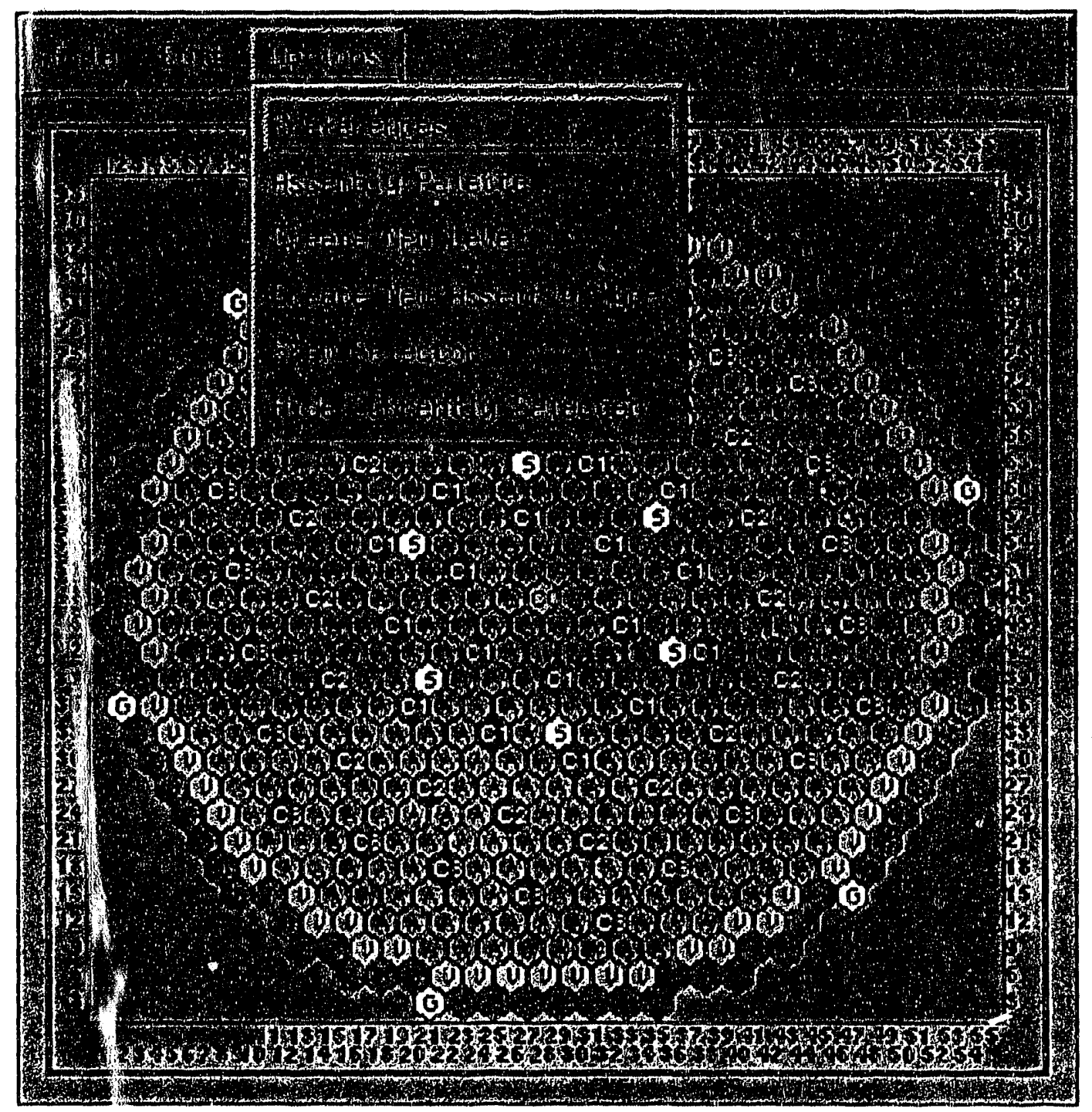

Figure 1. FaceMap Panel for the JOSHUA FM Function 
The Assembly Palette Panel is shown in Figure 2. The Assembly Palette contains a menu of assemblies that already exist, i.e., have already been created and for which complete named data records exist in the read-path hierarchy. The user selects assemblies from this list by clicking (with the mouse) on an assembly name in the Palette. The response to this select procedure depends on the state of the application. If positions in the reactor map were previously selected, then the assembly type from the Palette will be placed in each of those reactor facemap positions. If no facemap positions were selected, then the assembly from the Palette will be loaded into the Assembly Editor.

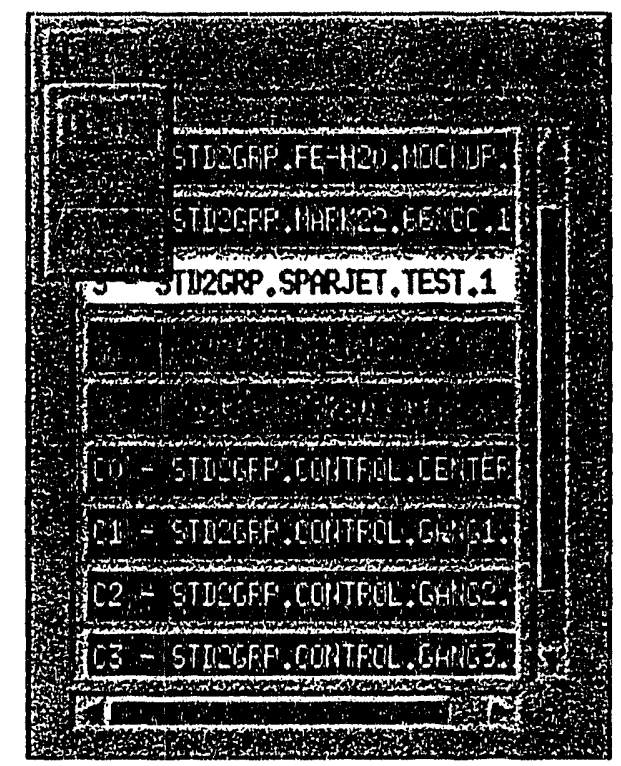

Figure 2. Assembly Palette Panel for JOSHUA FM Function

IV.2.2.3

Assembly Editor Panel

The Assembly Editor Panel, shown in Figure 3, is used to define (i.e., create and edit) assemblies.

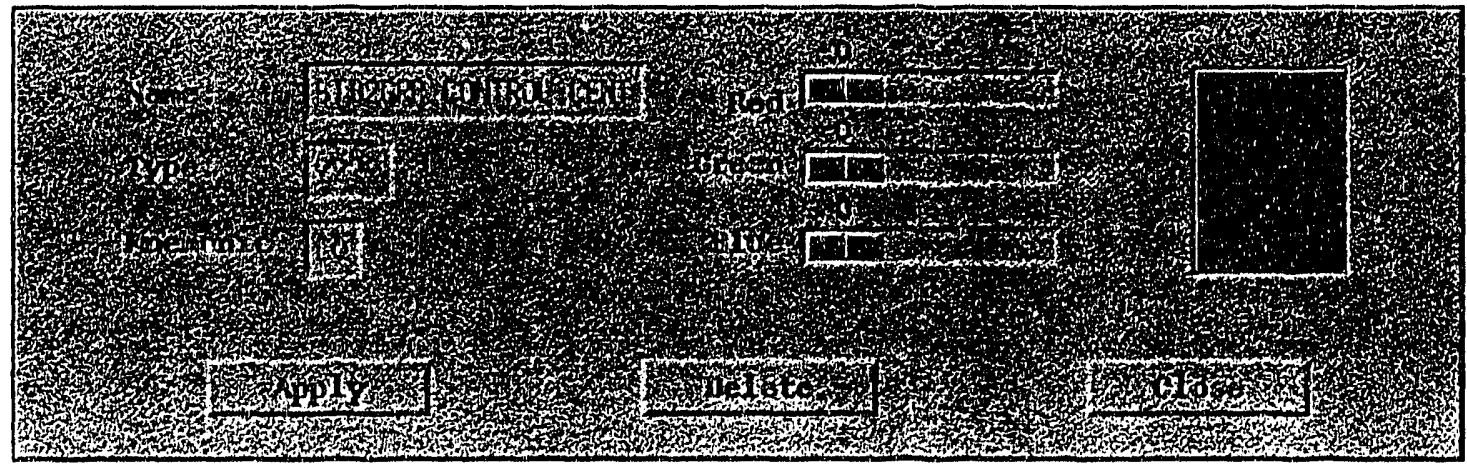

Figure 3. Assembly Editor Panel for JOSHUA FM Function 
An assembly definition consists of a name, type, mnemonic and color. The name identifies the desired GLASS record of cell averaged cross section data. The type and mnemonic are used only for compatibility with the current set of codes and make the FM function backwards compatible with existing RM-created records. The color permits the charge designer to graphically differentiate between assembly types. When the user creates an assembly type and presses the apply button, the assembly is placed in the Assembly Palette.

IV.2.2.4

Preferences Panel

The Preferences Panel, shown in Figure 4, is used to set the application display to the users choice.

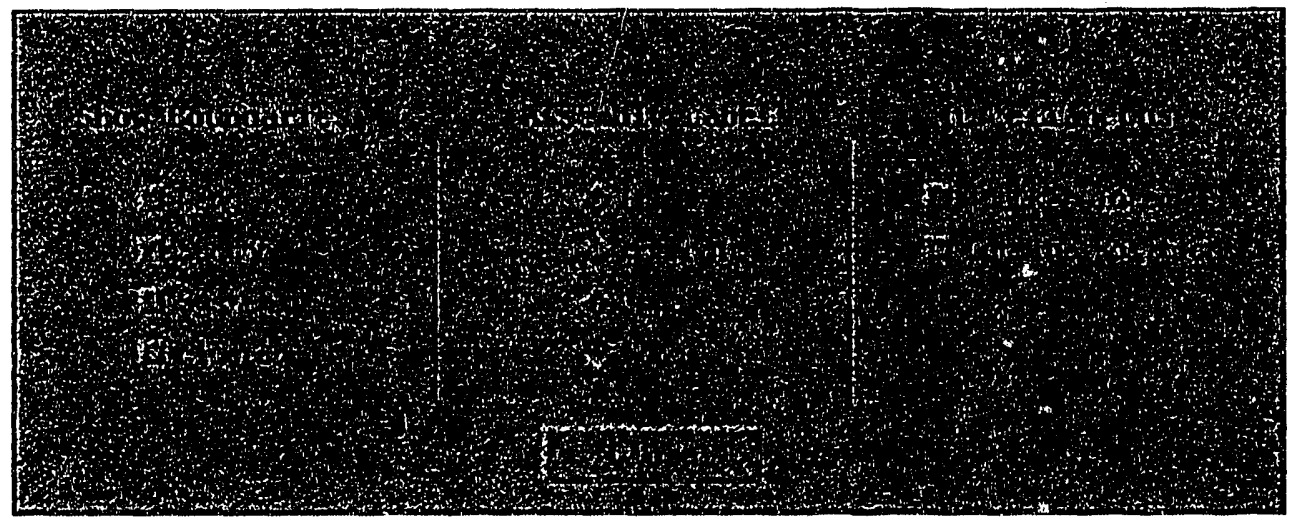

Figure 4. Preferences Panel for JOSHUA FM Function.

Sector, system, gang and cluster boundaries may be toggled and, when 'on', will be displayed on the FaceMap Panel as white lines. The positions of the FaceMap can be displayed with OLC\#, mnemonic, type or no label. Pointer tracking, a crosshair that follows the mouse and spans the length and width of the FaceMap Panel, can be toggled. A black outline can be placed on the positions of the FaceMap Panel to help differentiate two similar colored adjacent positions.

\section{IV.2.2.5}

Selector Panel

The Selector Panel, shown in Figure 5, is used to specify symmetry options and identify specific groupings and type of reactor positions. This panel consists of four small facemaps, each of which represents a specific grouping of reactor positions; namely, yangs, sectors, systems, clusters. The user selects a grouping by pressing the mouse button while the pointer is inside a particular grouping. To copy sector \#1 to sector \#3, the user 'selects' sector \#1 and chooses copy from the Edit menu on the FaceMap display. Then the user selects sector \#3 and chooses paste from the same Edit menu. Extended selections can be made by holding down the shift key while pressing the mouse button. 


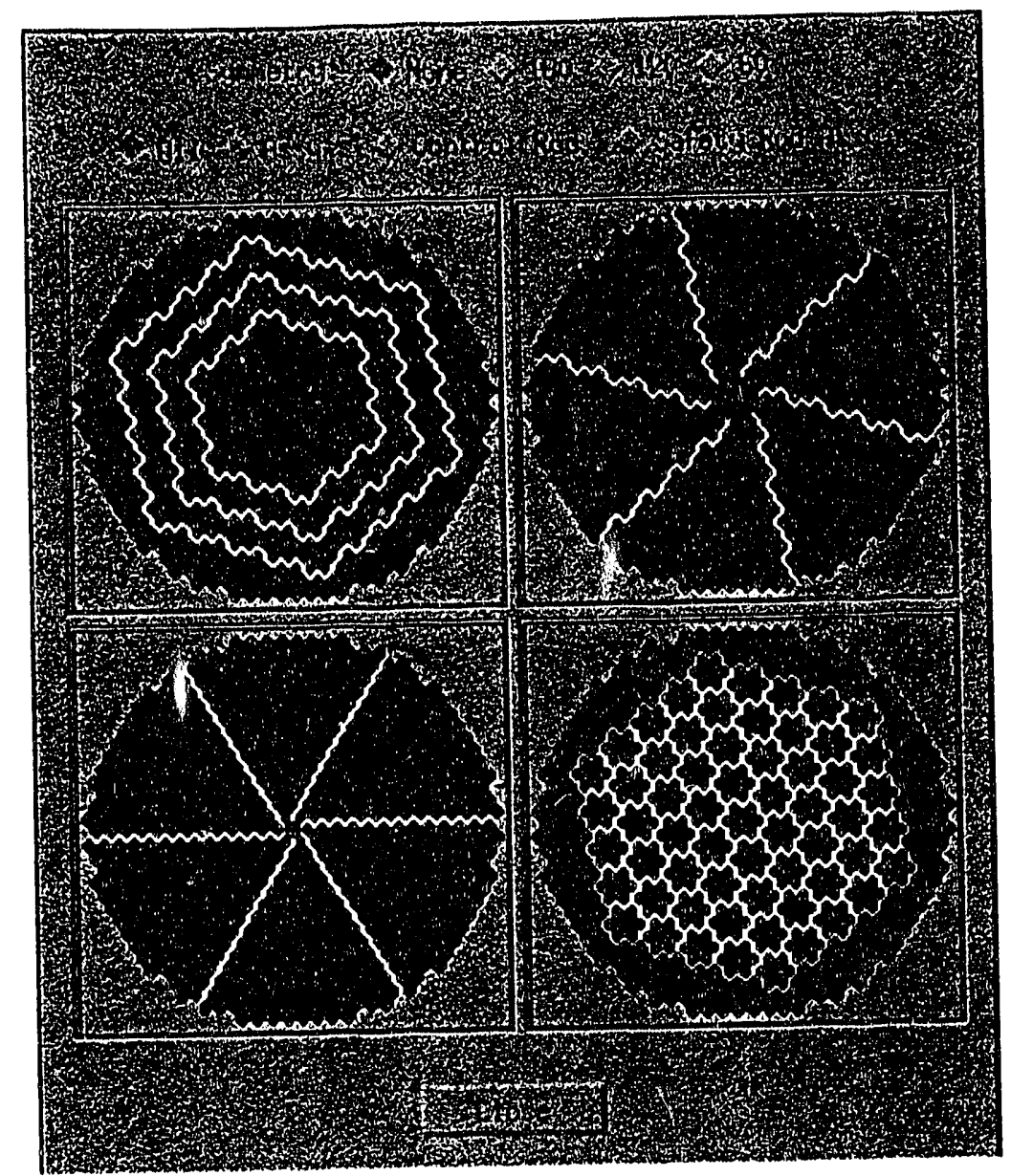

Figure 5. Selector Panel for JOSHUA FM Function

The selector also has two sets of toggle buttons which affect symmetry (e.g., 600, 1200, $180^{\circ}, 360^{\circ}$ ) and position type. The symmetry option changes the way selecting works. With $60^{\circ}$ symmetry a selection is replicated in each sector. The position type toggles affect which type of positions (assemblies, control rods, safety rod assemblies) are being referenced with the selector panel. For example, if gang 2 and the Control Rod toggle were selected then the positions referenced would be all the control rod positions in gang 2 .

\section{FUTUURE}

Consistent with ASCENT's charter, the Team will continue to focus on the following interconnected data management tasks: (a) 1/O data management or symbol manipulation, which comprises the interface between the user and the $1 / O$ data base; (b) computational data management, which comprises the interface between the numerically intensive part of the computer code and the corresponding computational, or 'internal', data base; and (c) interface, or flow of data, between the I/O data base and the computational data base. 

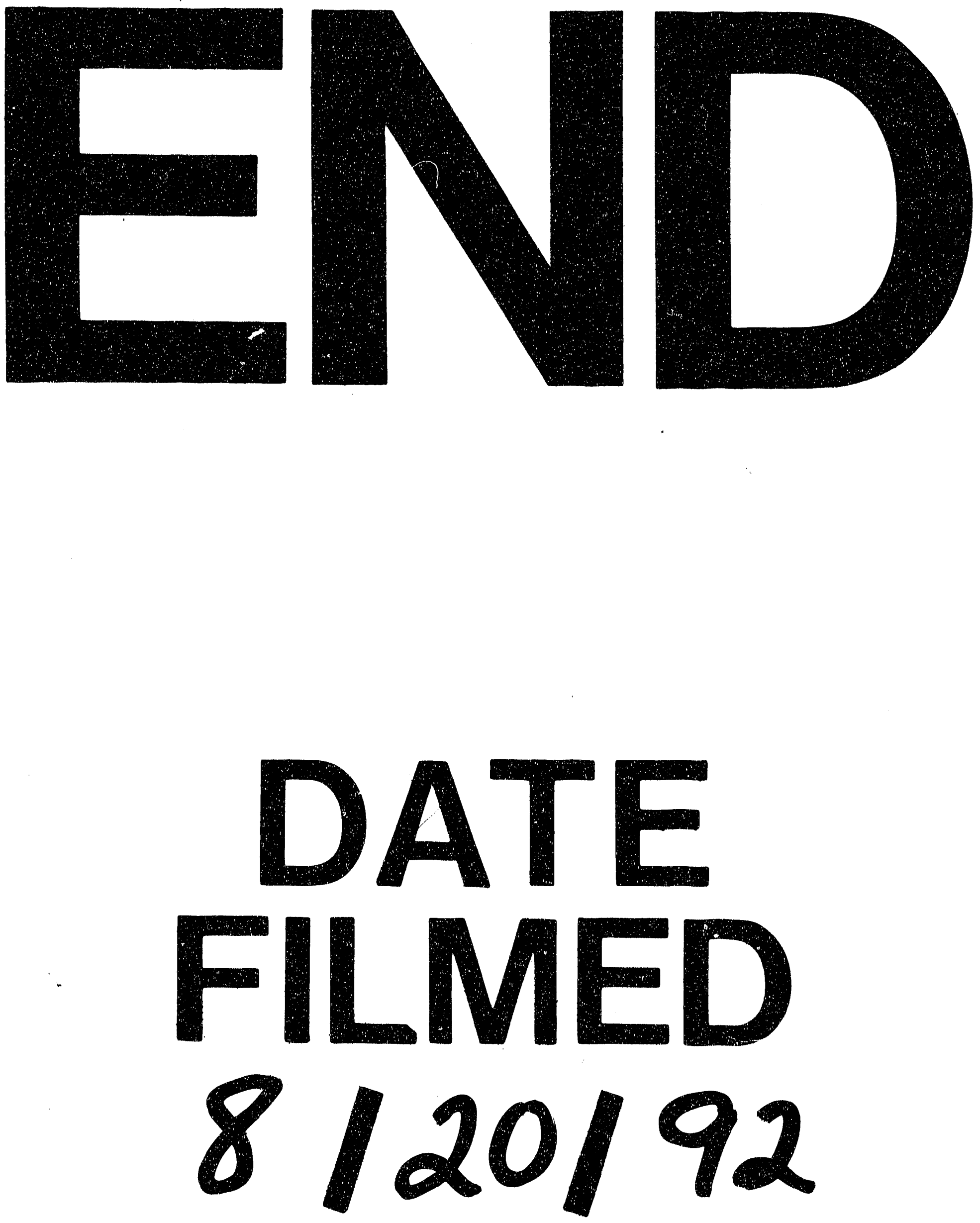
. . . . . 